\title{
Importancia clínica de la prueba calórica mínima
}

\section{Clinical importance of the minimal caloric test}

Héctor Riveros M', Gaudio Callejas C², Carlos Nieme³, Pilar Gajardo³, José Luis Anabalón4, Gaudio Correa4.

\section{RESUMEN}

Introducción: En la evaluación de la patología vestibular habitualmente se utiliza la prueba calórica clásica de Fitzgerald y Hallpike (POC). Sin embargo, esta sólo evalúa la función del nervio vestibular superior a través de la estimulación del canal semicircular lateral. La prueba calórica mínima (PCM) permite evaluar tanto la función del nervio vestibular superior como del nervio vestibular inferior a través de la estimulación del canal semicircular lateral y posterior respectivamente. Se desconoce el real impacto de la PCM en la evaluación clínica de pacientes con sintomatología vestibular.

Objetivo: Quantificar la utilidad diagnóstica de la POM en pacientes con sintomatología vestibular.

Material y método: Estudio diagnóstico transversal. Se obtuvo la sensibilidad y especificidad de la POM al comparar la respuesta del canal semicircular lateral en la POM con la prueba calórica convencional. Se evaluó en cuántos casos la información sobre la función del nervio vestibular inferior aportada por la POM fue relevante en el diagnóstico vestibular.

Resultados: Se estudió a 44 pacientes. Tanto la sensibilidad como la especificidad de la PCM para detectar patología al estimular el canal semicircular lateral fue de 93\% (IC95\%: 84\%-100\% y 80\%-100\% respectivamente). En 16\% ( $N=7$ ) de los casos la $P C M$ detectó patología del nervio vestibular inferior con POC normal.

Conclusiones: La POM es un examen complementario útil en la evaluación de pacientes con sintomatología vestibular, puesto que tiene una alta sensibilidad y especificidad para detectar patología del nervio vestibular superior a través de la estimulación del canal semicircular lateral y además, aporta información sobre el estado del nervio vestibular inferior a través de la estimulación del canal semicircular posterior, que no aporta la POC.

Palabras clave: Vértigo, prueba calórica mínima, prueba calórica.

\begin{abstract}
Introduction: The classic Fitzgerald-Hallpike caloric test (OCT) is usually utilized for evaluation of vestibular pathology. With this test, however, superior vestibular nerve function is assessed through stimulation of the lateral semicircular canal only. The minimal caloric test (MCT) allows for evaluation of both superior and inferior vestibular nerve function, through horizontal and posterior semicircular canals stimulation, respectively. Theactual impact of MCT on the clinical evaluation of patients presenting with vestibular symptoms is unknown.

Purpose: To quantify the diagnostic usefulness of MCT in patients with vestibular symptoms.

\footnotetext{
Profesor Auxiliar Asociado Departamento de Otorrinolaringología, Pontificia Universidad Católica de Chile.

Residente, Departamento Otorrinolaringología, Pontificia Universidad Católica de Chile.

Médico Grujano, Pontificia Universidad Católica de Chile.

Tecnólogo Médico, Departamento Otorrinolaringología, Pontificia Universidad Católica de Chile.
} 
Material and Method: Transversal diagnostic study. Sensitivity and specificity of MCT were obtained by comparing the lateral semicircular canal response in both MCT and the conventional caloric test. We assessed the number of cases in which the vestibular nerve function information obtained with MCT was relevant for vestibular diagnostic.

Results: 44 patients were studied. The sensitivity and specificity of MCT to detect pathologic conditions via lateral semicircular canal stimulation were 93\% (Cl 95\%: $84 \%-100 \%$ and $80 \%-100 \%$, respectively). In $16 \%(n=7)$ of the cases, MCT detected inferior vestibular nerve involvement with normal OCT.

Conclusions: MCT is a complementary test, which can be useful for evaluation of patients with vestibular symptoms, given its high sensitivity and specificity to detect superior vestibular nerve pathology through lateral semicircular canal stimulation. In addition, it provides information on the inferior vestibular nerve status, through posterior semicircular canal stimulation. The latter is not provided by conventional CCT.

Key words: Vertigo, minimal caloric test, classic caloric test, caloric test.

\section{INTRODUCCIÓN}

Generalmente se evalúa la sintomatología vestibular en forma inicial con un examen de VIII par que incluye la prueba calórica clásica de Ftzgerald y Hallpike ${ }^{1}$ (POC) descrita en 1942. Sin embargo, en ocasiones esta evaluación inicial no encuentra alteraciones pese al claro relato de sensación de giro del paciente. Lo anterior podría deberse a que la POC está diseñada para evaluar la función del nervio vestibular superior a través de la estimulación del canal semicircular lateral (CSC), pero no evalúa la función del nervio vestibular inferior.

A través de los años sehan diseñado otras pruebas para evaluar la función vestibular, entre ellas la prueba calórica mínima ${ }^{2-4}$ (POM), diseñada para evaluar tanto el nervio vestibular superior (através delaestimulación del CSO) como el nervio vestibular inferior a través de la estimulación del canal semicircular posterior (CSCP). La POM se basa en el hecho que pequeños volúmenes de agua son suficientes para estimular el aparato vestibular y qué cambios de posición de la cabeza permiten observar la respuesta del CSO y, luego, del CSCP. Laestimulación del CSO_ selogracon lacabeza extendidaen $60^{\circ}$, en cambio, la estimulación del CSOP se consigue con la cabeza en anteflexión en $30^{\circ}$, ya que en esta posición el CSO se encuentra en posición neutra, lo que impide su estimulación. Las ventajas de la POM son la capacidad de investigar ambos nervios vestibulares, un relativo corto tiempo parasu realización y unamenor incidenciade síntomas neurovegetativos. Por otro lado, una desventaja es que no permite el estudio de la dirección preponderante.
Existen estudios sobre la utilidad de la POM en el diagnóstico diferencial de pacientes con sintomatología vestibular cuando la POC no explica plenamente la sintomatología del pacientes. Sin embargo, según nuestro conocimiento no hay estudios que analicen su relevancia en términos de sensibilidad y especificidad ni que cuantifiquen su aporte a la POCen la evaluación de pacientes con sintomatología vestibular.

\section{OBJETIVO}

日 objetivo de este trabajo es cuantificar la utilidad diagnóstica de la POM en pacientes con sintomatología vestibular. Para esto se obtuvo la sensibilidad y especificidad de la POM para detectar patologíaal estimular el canal semicircular lateral al compararla con la prueba calórica convencional (POC) y se cuantificó su utilidad como complemento de la POC al aportar información sobre la función del nervio vestibular inferior.

\section{MATERIAL Y MÉTODO}

En forma prospectiva se realizó un estudio transversal en el Departamento de Aorrinolaringología de la Pontificia Universidad Católica de Chile, entre agosto de2005 y marzo de2006. Sesolicitó POM a todos los pacientes derivados por el mismo otorrinolaringólogo para la realización de un VII par debido a sintomatología vertiginosa Se obtuvo la sensibilidad y especificidad de la POM al comparar la respuesta del canal semicircular lateral en la POM con la prueba 
calórica convencional (gold standard). Luego se eva luó en cuántos casos la POM aportaba información relevante (respuesta del canal semicircular posterior alterada con PCC normal) para el diagnóstico vestibular. Todos los exámenes fueron realizados por dos tecnólogos médicos de nuestra unidad entrena dos en las técnicas de las pruebas.

La prueba calórica mínima se realizó de la siguiente manera: Con el paciente sentado con el oído a examinar hacia arriba, se instila $2 \mathrm{cc}$ de agua a $2^{\circ} \mathrm{C}$, después de 20 segundos, se extrae el agua poniendo al paciente en posición opuestay luego, con la cabeza en extensión en $60^{\circ}$, se busca nistagmus horizontal por estimulación del canal semicircular lateral. A cesar el nistagmus, se lleva la cabeza a anteflexión en $30^{\circ}$ y se busca nistagmus rotatorio por estimulación del canal semicircular posterior. 1 mismo procedimiento se repite en el oído contralateral cinco minutos después. La prueba calórica clásica se realizó según lo descrito por Ftzgerald y Hallpike'.

Los criterios diagnósticos fueron los siguientes: VIII par (gold standard):

- Consideramos hipofunción vestibular unilateral una diferencia entre las respuestas de ambos lados mayor a 20 segundos. Al menos un lado debía presentar una respuesta mayor a 1 minuto 30 segundos.

- Consideramos hipofunción vestibular bilateral una respuesta menor a 1 minuto 30 segundos en ambos lados.

PCM:

- Consideramos hipofunción vestibular unilateral una diferencia entre las respuesta de los canales semicirculares respectivos de ambos lados mayor o igual a 15 segundos.

- Consideramos hipofunción vestibular bilateral en forma cualitativa.

\section{RESULTADOS}

Durante el periodo de tiempo comprendido entre agosto de 2005 y marzo de 2006 se estudió a 44 pacientes con un promedio de edad de 55 (13 a 93) años, $43 \%(n=19)$ hombres y $57 \%(n=25)$ mujeres (Figura 1).

\section{Sensibilidad y especificidad}

La sensibilidad y especificidad de la POM para detectar patología al estimular el canal semicircular lateral fue de $93 \%$ (IC95\%: $84 \%-100 \%$ y $80 \%-100 \%$, respectivamente). Los datos se resumen en la Figura 2.

\section{Utilidad de la prueba calórica mínima como complemento de la prueba calórica convencional}

En 16\% ( $N=7)$ del total de pacientes la POM detectó alteración al estimular el canal semicircular posterior cuando la PCC fue normal. La Figura 3 muestra un diagrama de flujo que resume la información.

\section{DISCUSIÓN}

En los 44 pacientes con sintomatología vertiginosa evaluados encontramos una sensibilidad y especificidad de la PCM para detectar patología al estimular el canal semicircular lateral de 93\% (IC95\%: $84 \%-100 \%$ y $80 \%-100 \%$, respectivamente). En $16 \%(\mathrm{~N}=7)$ de los casos la PCM fue fundamental para el diagnóstico, detectando patología del nervio vestibular inferior (a través de la estimulación del CSCP) cuando la PCC fue normal.

Un paciente presentó PCM alterada para el CSCL con POC normal (falso positivo). Creemos

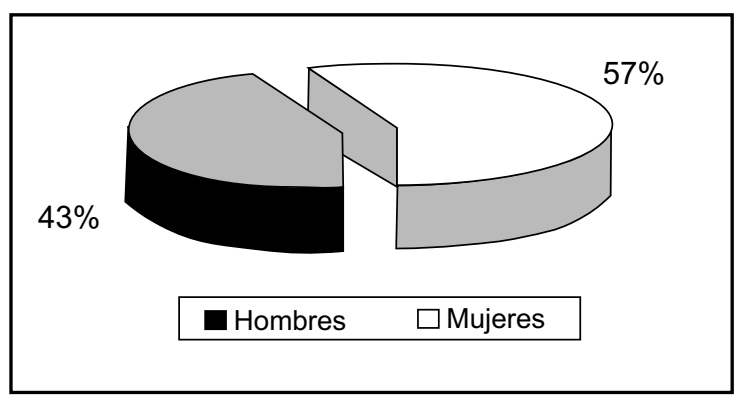

Figura 1. Distribución de la muestra por sexo. 


\begin{tabular}{|l|c|c|}
\hline \multirow{2}{*}{ PCM (cscl) } & \multicolumn{2}{|c|}{ PCC } \\
\cline { 2 - 3 } & alterado & normal \\
\hline alterada & 27 & 1 \\
\hline normal & 2 & 14 \\
\hline \multirow{2}{*}{} & 0,93 & 0,93 \\
& $\left(\mathrm{IC}_{95 \%}: 84 \%-100 \%\right)$ & $\left(\mathrm{IC}_{95 \%}: 80 \%-100 \%\right)$ \\
\cline { 2 - 3 } & sensibilidad & especificidad \\
\hline \multirow{2}{*}{ PCM: prueba calórica mínima. cscl: canal semicircular lateral. PCC: prueba calórica convencional. }
\end{tabular}

Figura2. Sensibilidad y especificidad al comparar la respuesta del canal semicircular lateral en la prueba calórica mínima con la prueba calórica convencional.

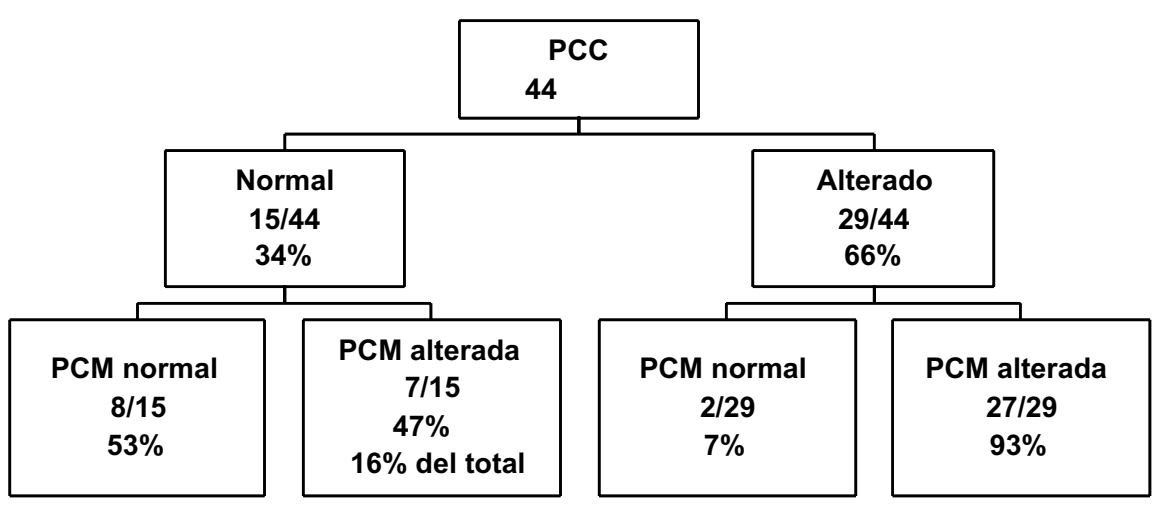

Figura 3. Diagrama de flujo de la evaluación complementaria entre prueba calórica convencional y prueba calórica mínima.

que esto podría deberse a que la PCM se realiza con un volumen muy pequeño de agua $(2 \mathrm{cc})$ y que por lo tanto factores como la conformación del CAE o la densidad de pelos podrían afectar la interacción del agua con la membrana timpánica alterando el resultado de la prueba.

Hay autores que refieren que la PCM puede llegar a ser dolorosa, sin embargo, ninguno de nuestros pacientes experimentó dolor y sólo algunos presentaron molestias tolerables.

Vale la pena mencionar que al evaluar la respuesta del canal semicircular posterior en la PCM (con la cabeza en anteflexión de $30^{\circ}$ ) podría también estar estimulándose en menor grado el canal semicircular superior, sin embargo, esto no nos parece de gran relevancia puesto que este último canal es inervado por el nervio vestibular superior que ya es evaluado al estimular el canal semicircular lateral.

Como ya se describió en una publicación anterior $^{5}$ la PCM tendría útiles aplicaciones, como son: 1) Para continuar con la evaluación de un síndrome vertiginoso con POCnormal. 2) En la evaluación de un paciente con parálisis vestibular en la PCC y síndrome vertiginoso de mayor duración a lo esperado según la compensación central. 3) Distinguir la patología del nervio vestibular superior de la del inferior. Este último punto puede ser de gran utilidad en la evaluación de neurinomas permitiendo determinar cuál es el nervio comprometido en neurinomas intracanaliculares pequeños.

Una limitación de nuestro estudio es que el mismo tecnólogo médico realizó ambos exámenes (PCM y PCC) a cada paciente. Aunque hubo varios 
días de diferencia entre cada examen, este hecho va en desmedro del ciego. Oreemos que sería útil repetir la experiencia mejorando este punto, para lo cual cada examen (PCM y PCC) debería ser realizado por un tecnólogo médico diferente que desconociera el resultado del otro examen. Por otra parte, otra limitación pareciera ser un tamaño muestral pequeño de 44, sin embargo, los intervalos de confianza para los valores de sensibilidad y especificidad calculados fueron aceptables (84\%$100 \%$ y $80 \%-100 \%$, respectivamente).

En conclusión nos parece que la PCM es un examen útil en la evaluación de pacientes con sintomatología vestibular, puesto que tiene una alta sensibilidad y especificidad para detectar patología del nervio vestibular superior (al estimular el canal semicircular lateral) y además, aporta información sobre la función del nervio vestibular inferior (a través de la estimulación del canal semicircular posterior) que no aporta la PCC. Oreemos que es de gran utilidad tener presente este examen como complemento a la PCC en la evaluación de pacientes con sintomatología vestibular, especialmente cuando la evaluación inicial con PCC no detecta patología.

\section{BIBLIOGRAFÍA}

1. Ftzgrand G Halpike $C$. Studies in human vestibular function: Cbservations on the directional preponderance of caloric nystagmus resulting from cerebral lesions. Brain 1942; 65: 115.

2. LINTHIOUM F, OHURCHILL D. Vestibular tests results in acoustic tumor cases. Arch Aolaryng 1968; 88: 604-7.

3. LINTHIOM F, WALDORF R, LUXFORD W. Infrared/ videorecording of rotatory nystagmus arising from the posterior semicircular canal via the singular nerve. Seminars in hearing 1989; 10: 191-5.

4. JaCOBSON Ex AL. A reappraisal of the monothermal warm caloric screening test. Ann Otol Rhinol Laryngol 1995; 104: 942-5.

5. Riveros H, Comen M, Badía P, Anabalón Jl, Correa C. Utilidad de la prueba calórica mínima. Rev Atorrinolaringol Cir Cabeza Ouello 2005; 65: 193-6. 